\title{
IR Dual-Band Camera Demonstrator and Dual-Band Image Fusion Techniques
}

\author{
Adomeit, Uwe \\ FGAN-FOM Research Institute for Optronics and Pattern Recognition \\ Gutleuthausstr. 1, D-76275 Ettlingen, Germany
}

\section{INTRODUCTION}

During the last decades, thermal imagers have evolved from "simple" scanning systems to state-of-the-art focal plane arrays (FPA). Nowadays, different trends of development can be seen, for example decrease in pixel size, new materials (InAs/GaSb superlattices) and especially multispectral imagers. A multispectral imager is able to acquire information in different channels of the electromagnetic spectrum at the same time, while the standard broadband systems only work in one channel of the electromagnetic spectrum. One type of multispectral imager is the so-called Dual-Band Camera which typically acquires images simultaneously in the $8-12 \mu \mathrm{m}$ (LWIR) and 3-5 $\mu \mathrm{m}$ (MWIR) spectral range. Advantages foreseen for these cameras are manifold. They range from simply using the pros and cons of both bands at the right time for automatic and human target recognition due to specific emissivity features. In Germany, AIM INFRAROT-MODULE GmbH in co-operation with the Fraunhofer IAF (Institute for Applied Solid State Physics) developed a Dual-Band Detector based on QWIP technology [1, 2]. This detectorcooler-assembly was made available to FGAN-FOM for system integration which was conducted in cooperation with IRCAM GmbH. The necessary special IR Dual-Band Optics was developed in co-operation of FGAN-FOM and Opto-System-Technik. All components together resulted in the IR Dual-Band Camera demonstrator which was then experimentally assessed in the thermal imager laboratory of FGAN-FOM. Additionally this demonstrator was used to test dual-band image fusion techniques in the laboratory as well as in field trials.

\section{THE IR DUAL-BAND CAMERA DEMONSTRATOR}

The IR-Dual-Band Camera demonstrator is based on a cooled QWIP FPA detector with $384 \times 288$ detector elements of $40 \mu \mathrm{m}$ pitch. It is operating simultaneously in the spectral range of $4.3 \mu \mathrm{m}$ to $5.3 \mu \mathrm{m}$ (in the following: MWIR) and $7.7 \mu \mathrm{m}$ to $8.7 \mu \mathrm{m}$ (in the following: LWIR), pixels for the two spectral ranges being stacked one upon the other [2]. So the acquisition occurs simultaneous spatially as well as temporally. This is the ideal acquisition for a multispectral system from the signal processing point of view.

Two optics were specially developed for the demonstrator. The first optic consists of a mirror/lens combination for a dual field of view. Its construction was laid out to assure a stable focus for both bands also under changing thermal conditions. Focal lengths are $86 \mathrm{~mm}$ for the Wide Field of View (WFOV) and $390 \mathrm{~mm}$ for the Narrow Field of View (NFOV). The resulting fields of view (FOV) are $10.2^{\circ} \times 7.7^{\circ}$ and $2.3^{\circ} \times 1.7^{\circ}$ respectively, F-number is two for both fields of view. The second optic is a standard $100 \mathrm{~mm}$ $\mathrm{F} / 2$ refractive system equipped with an anti reflective coating suited for both spectral ranges and results in a FOV of $8.8^{\circ} \times 6.6^{\circ}$.

Image acquisition is carried out with 14-bit depth and up to $300 \mathrm{~Hz}$ frame rate. The integration time depends on the frame rate. Typically a frame rate of $100 \mathrm{~Hz}$ is used which corresponds to an integration time of $6.8 \mathrm{~ms}$. The acquisition soft- and hardware allows the loss-free image acquisition up to the maximum frame rate. It shows both bands simultaneously and additionally allows the superposition of the two bands in real time false color presentation.

\section{EXPERIMENTAL ASSESSMENT}

Up to now no commonly agreed rules for the experimental assessment of multispectral imagers exist. Based on experience with such systems it is recommended to use the figures of merit for broadband thermal imagers and to conduct three additional measurements with regard to the spectral crosstalk, the temporal synchronization and the spatial pixel alignment [3]. In the following examples are presented for experimental results on Spectral Response, System Transfer Function (SiTF), temporal and spatial Noise (Noise Equivalent Temperature Difference, NETD, and Inhomogeneity Equivalent Temperature Difference, IETD), and Modulation Transfer Function (MTF). For all measurements presented here, the $100 \mathrm{~mm} \mathrm{~F} / 2$ optics and an integration time of $6.8 \mathrm{~ms}$ corresponding to $100 \mathrm{~Hz}$ frame rate were used. 


\subsection{Spectral Response and Spectral Crosstalk}

The spectral response of the demonstrator was measured using equipment developed by FGAN-FOM. This equipment is based on a glow bar as radiation source, an integrating sphere to achieve a homogeneous radiation and narrow filters for the spectral selection. It results in the normalized spectral response as presented in Figure 1.

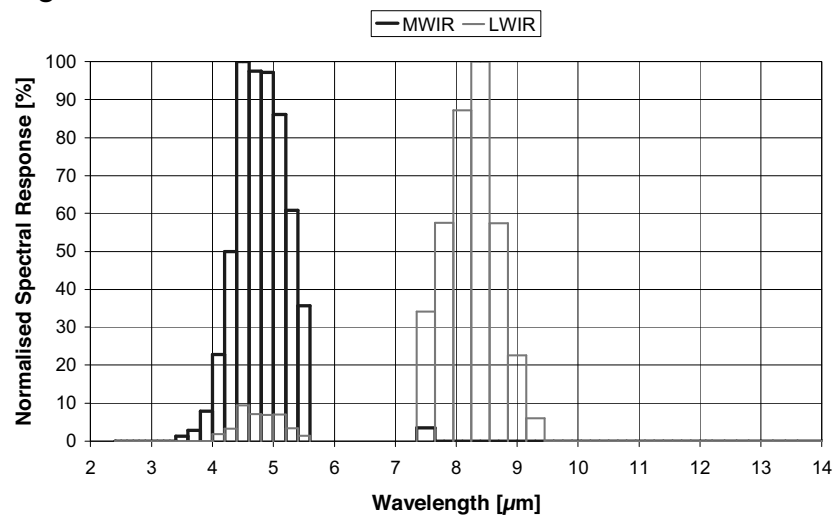

Fig. 1: Normalized spectral response for the two bands of the Dual-Band Camera demonstrator.

Based on these measurements, the central wavelengths were found to be $4.8 \mu \mathrm{m}$ and $8.2 \mu \mathrm{m}$ respectively. The full width at half maximum is approximately $1 \mu \mathrm{m}$ for both bands, so the overall spectral behaviour corresponds to the typical QWIP characteristics. From the data in Fig. 1 it is obvious that the LWIR band has some response in the MWIR, whereas it is not vice versa. The contribution of the spectral crosstalk to the response in the LWIR band is approximately $7 \%$. A reason for this crosstalk has not been determined yet, but up till now it is also unknown how such a crosstalk influences possible applications [3].

\subsection{System Transfer Function (SiTF) and Noise}

The SiTF describes the system response in form of output signal (here: digital levels, DL) as function of input signal (here: temperature). The responsivity is the slope of the SiTF at a given scene temperature. It is calculated by deviation of the SiTF at this temperature.

For measuring of the SiTF a black-body was placed in front of the optics, covering it fully. Using the digital output, image sequences were recorded in the temperature range from $5{ }^{\circ} \mathrm{C}$ to $75{ }^{\circ} \mathrm{C}$ with a step of $5 \mathrm{~K}$ and an additional measurement at $26.85^{\circ} \mathrm{C}(300 \mathrm{~K})$. These image sequences were averaged and then the average signal in dependence of scene temperature was calculated. From this data the responsivity is calculated for a scene temperature of $300 \mathrm{~K}$. The temporal noise is determined for $300 \mathrm{~K}$ scene temperature using the data acquired during the SiTF measurement. It is calculated separately for each pixel in the time domain and then averaged over all pixels excluding bad ones. The ratio calculation between temporal noise and responsivity results in the Noise Equivalent Temperature Difference (NETD). The Fixed Pattern Noise (FPN) is calculated in dependence of scene temperature, again using the data acquired during the SiTF measurement. First, the image sequences were averaged in order to remove the temporal noise. Then the standard deviation of the signal was calculated using a high pass filter. As the FPN depends on the scene temperature it was averaged in the temperature range from $10^{\circ} \mathrm{C}$ to $45^{\circ} \mathrm{C}$ for comparison.

As shown in Figure 2, the behaviour is different for the two bands. For most temperatures, the MW signal is lower than the LW signal. Also, the LWIR-SiTF is more linear than the MWIR-SiTF, a typical feature already known from single band QWIPs and a behaviour that should be considered when signal (twoband) processing is applied.

Table 1 shows the response to be approximately a factor of two higher in the MWIR than in the LWIR. As the temporal noise is slightly higher in the MWIR then in the LWIR, the NETD in the MWIR is only approximately a factor of 1.75 better compared to the LWIR. In spite of these differences the bands are very close together in their performance. The IETD is approximately the same for both spectral ranges. Although not perfect, the differences between the MWIR and the LWIR are within a magnitude that not automatically favors the MWIR spectral range. This is advantageous for signal processing as well as for practical use. For example the selection of the best band is not limited by the camera performance as had been the case in all prior dual-band cameras we investigated in the past. 


\begin{tabular}{|l|c|c|}
\hline & MWIR & LWIR \\
\hline Response at 300 K & $58 \mathrm{DL} / \mathrm{K}$ & $29 \mathrm{DL} / \mathrm{K}$ \\
\hline Temporal Noise at 300 K & $1.9 \mathrm{DL}$ & $1.7 \mathrm{DL}$ \\
\hline NETD at 300 K & $32 \mathrm{mK}$ & $56 \mathrm{mK}$ \\
\hline Fixed Pattern Noise $\left(\mathbf{1 0}^{\circ} \mathbf{C}-\mathbf{4 5}^{\circ} \mathbf{C}\right)$ & $1.6 \mathrm{DL}$ & $1.3 \mathrm{DL}$ \\
\hline IETD $\left(\mathbf{1 0}^{\circ} \mathbf{C}-\mathbf{4 5}^{\circ} \mathbf{C}\right)$ & $27 \mathrm{mk}$ & $23 \mathrm{mK}$ \\
\hline
\end{tabular}

Table 1: Performance data of the Dual-Band Camera demonstrator for both spectral ranges. The values were measured with $100 \mathrm{~mm} \mathrm{~F} / 2$ optics at $100 \mathrm{~Hz}$ frame rate and $6.8 \mathrm{~ms}$ integration time respectively (DL: digital levels).

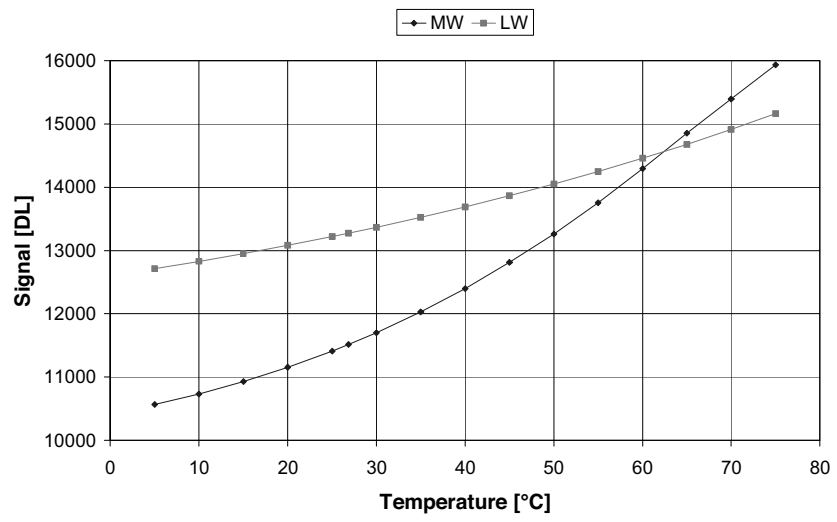

Fig. 2: SiTF of the Dual-Band Camera demonstrator for both spectral ranges measured with $100 \mathrm{~mm} \mathrm{~F} / 2$ optics at $100 \mathrm{~Hz}$ frame rate and $6.8 \mathrm{~ms}$ integration time (DL: digital levels).

\subsection{Modulation Transfer Function and Spatial Alignment}

The Modulation Transfer Function (MTF) is the parameter that describes both the spatial resolution and image quality of an imaging system in terms of spatial frequency response. The MTF concept is not suited to assess the spatial transfer characteristics of an advanced thermal imager (e. g. Focal Plane Arrays) as a whole. Here, a separation in prefilter MTF (all components that act on the signal before sampling, e. g. front optics, detector etc.) and postfilter MTF (all components that act on the signal after sampling, e. g. electronics, video display etc.) is necessary [4]. As the measurements for the Dual-BandCamera demonstrator were done using the digital signal, the measured MTF corresponds to the prefilter MTF. Different methods for measuring the MTF are known. The one applied here is the so-called scanning edge method. For this method, an edge image is moved in small steps over the detector mosaic and the signal of one detector element is measured in dependence of edge position. The resulting edge function is deviated to get the Line Spread Function (LSF) from which the MTF is derived by Fourier transformation.

$\rightarrow$ MWIR $\rightarrow-$ LWIR

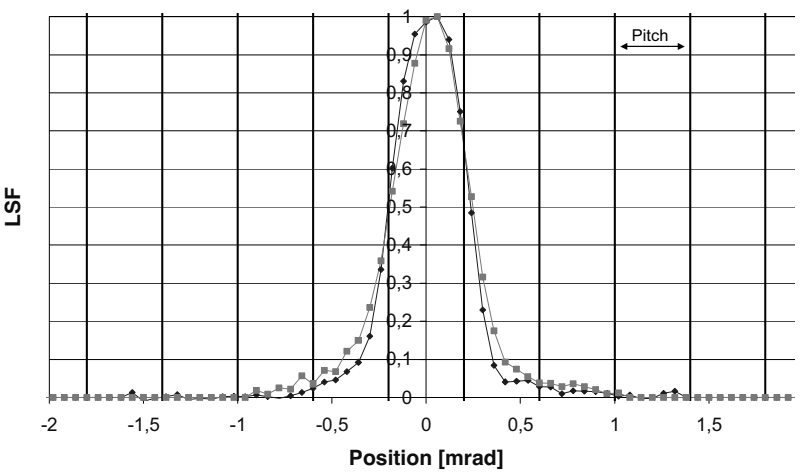

$\rightarrow-M W I R \rightarrow-$ LWIR

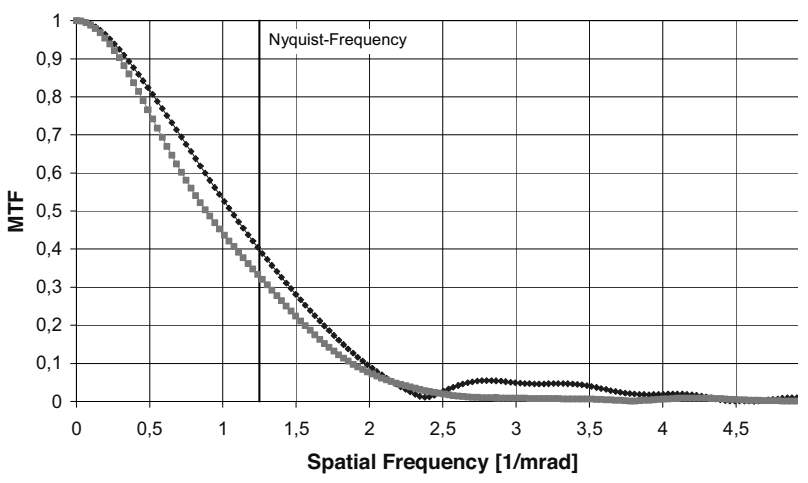

Fig. 3: LSF (left) and MTF (right) of the Dual-Band Camera demonstrator measured horizontally when equipped with the $100 \mathrm{~mm} \mathrm{~F} / 2$ Dual-Band Optics. Presented are both spectral ranges. Additionally pitch and Nyquist-Frequency are given within the graphs.

Figure 3 shows LSF and MTF of the IR-Dual-Band-Camera demonstrator when equipped with $100 \mathrm{~mm}$ F/2 optics for both spectral ranges and horizontal measurement. It shows LSF and MTF to be 
independent from the spectral range and this holds true for also for vertical measurement. The LSF is atypically narrow for a QWIP detector, resulting in an undersampled MTF. It can thus be concluded, that the methods used to improve the detector MTF [1] were successful.

LSF and MTF automatically give a statement about the spatial alignment of a multispectral system if the measurement is conducted at the same time in all spectral channels [3]. As this was done here, it can be concluded that a perfect spatial alignment is given and thus superimposition of the bands is possible without disturbing effects. This conclusion is possible because a misalignment would result in differences in LSF and/or MTF of the two bands [3].

\section{DUAL-BAND IMAGE FUSION TECHNIQUES}

Dual-Band image fusion techniques were studied at FGAN-FOM for some time already before the IR Dual-Band Camera demonstrator was available. They were based i. e. on single band MWIR and LWIR QWIP cameras. The problem with a two camera approach is that a lot of effort goes into pixel registration, a problem that vanishes with a true Dual-Band System.

An often used approach for multispectral image fusion and one also available in the software of the demonstrator is color fusion. Here, for each band a different color (for example red and blue for two bands) is used, resulting in a false color presentation of the fused information. The problem associated with this approach is the dependence of the resulting image from the scaling (gain and offset) applied to convert the information into 8-bit for presentation. Examples for this technique can be found in the literature $[6,7]$.

Another approach is the measurement of the absolute temperature based on the ratio principle [8-10]. Although this method looks very promising, it is problematic for low scene temperature applications because the reflective radiation component is neglected.

We developed a novel method in order to make optimum use of the differences of the two bands in amonochrome monitor giving specific enhanced contrasts to objects of interest. The basic idea is simple and makes use of a band dependence on an object parameter (emissivity etc.). In order to extract these differences, the bands have to be adapted somehow because the SiTF are different (compare Figure 2). If the bands are adapted, ratio or subtraction reveals the differences. As there is a complex behaviour of emission, reflection, transmission and path radiance in both bands it is in most cases difficult or even impossible to extract the exact physical origin for a signal difference. The approach was to calibrate the two bands in apparent temperatures ( $\left.T_{\text {app, LW }} ; T_{\text {app,Mw }}\right)$ and then calculate the ratio $\left(R_{T}\right)$ or the difference $\left(D_{T}\right)$ of the apparent temperature :

$$
\begin{gathered}
R_{T}=\frac{T_{a p p, L W}}{T_{a p p, M W}} \\
D_{T}=T_{a p p, L W}-T_{a p p, M W}
\end{gathered}
$$

For calibration, a method was developed that uses two reference images at known temperatures and the once measured SiTF of the two bands [11].

An example for the method is shown in Figure 4. It shows a cup filled with hot water in front of a panel with printed bar patterns. The cup itself is also printed with some kind of advertisement. The scene was recorded with the IR Dual-Band Camera demonstrator and the acquired data were calibrated in apparent temperatures. In Figure 4 this calibrated data are presented using the same temperature range for both spectral ranges. With the standard linear scaling it is impossible to show the bar pattern as well as the printing on the cup at the same time. So, the data are shown with two different temperature ranges. The left image shows the printing on the cup, which is more prominent in the LWIR, whereas there is no contrast in the background. The right image shows the bar pattern, whereas the cup is saturated. Here the reflection of the hot cup on the panel in the background is obvious. After calculating the fused image, the bar pattern as well as the printing on the cup show up within the same image. In the example of Figure 4 it makes no difference whether the ratio or the difference of the apparent temperatures is calculated. We prefer the difference as the temperature difference gives a magnitude of the difference in the two bands and this may be usable to quantify spectral features.

Two more experimental images are presented in Figure 5 and 6 . The first image (Figure 5) shows a car that was parked in front of the institute. Whereas the two single band images showed nothing special, the fused image reveals writing on the rear window. The image also gives the impression that the license plate would be readable if the camera had a better spatial resolution. Figure 6 presents the two-band images of a truck with running motor. The exhaust of the truck is covered behind a low emissive material. When the image fusion is conducted, the low emissive material shows up as bright spot and is clearly detectable. Additionally, some other spectral effects are also visible on the street and the windows. 


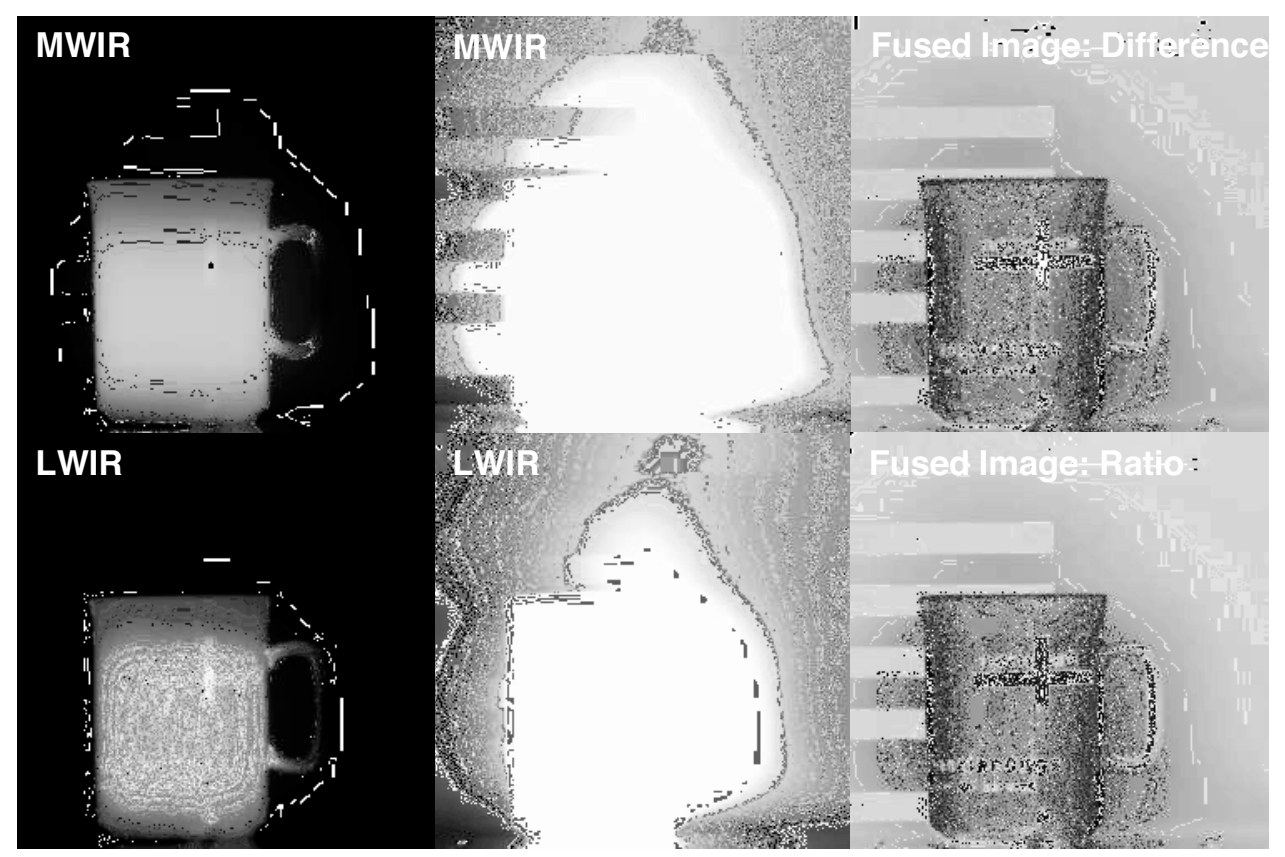

Fig. 4: Test image of a cup filled with hot water in front of a panel with bar targets. Presented are the two apparent temperature calibrated original images and the fused image. The fused image shows the difference in the apparent temperature of the two bands (upper image) and the ratio of the apparent temperature (lower image). The original images are displayed using the same temperature range for the two bands but with two different temperature ranges to show different details.

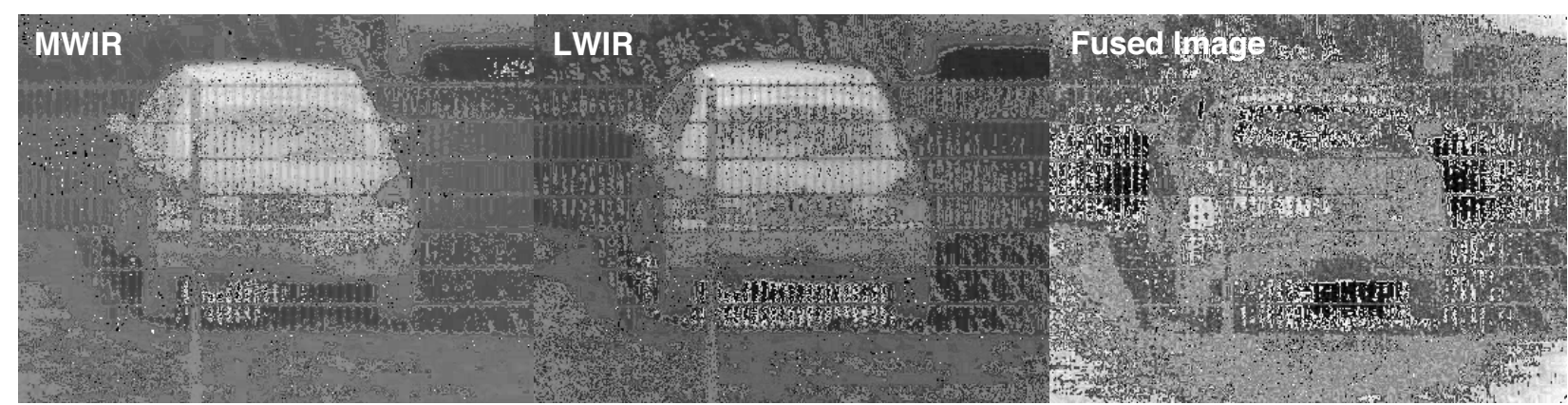

Fig. 5: Car with writing on the rear window recorded with the IR Dual-Band Camera demonstrator. Presented are the two apparent temperature calibrated original images and the fused image. The fused image shows the difference in the apparent temperature of the two bands. The original images are displayed using the same temperature range for both bands.

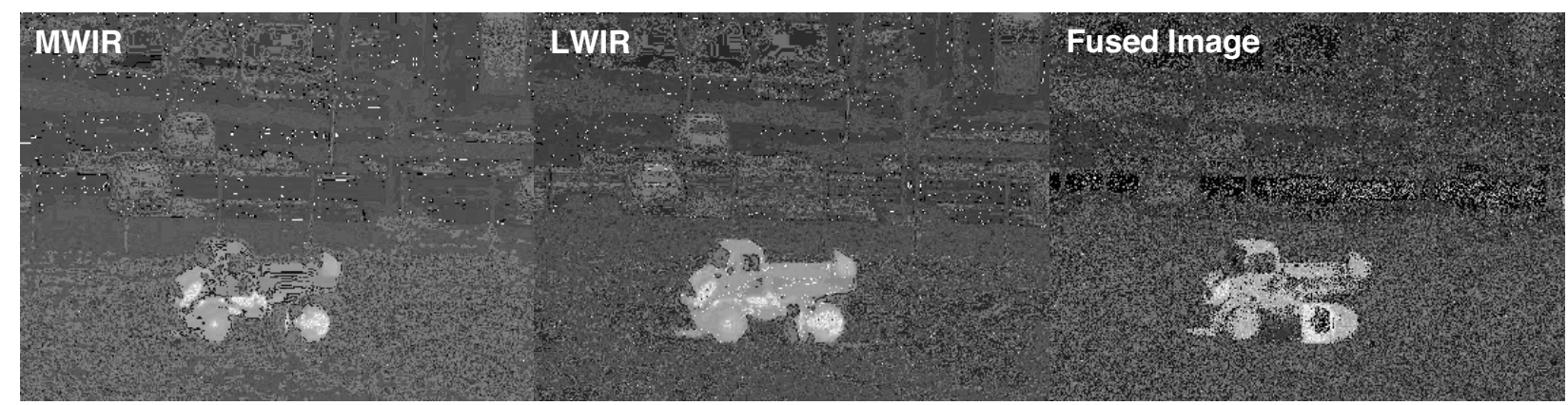

Fig. 9: Truck with running motor and low emissive material in front of the exhaust. Presented are the two apparent temperature calibrated original images and the fused image. The fused image shows the difference in the apparent temperature of the two bands. The original images are displayed using the same temperature range for both bands. Note the enhanced contrast of the background perceived by an observer. 


\section{SUMMARY AND CONCLUSIONS}

The IR Dual-Band Camera demonstrator was built by four partners and FGAN-FOM. It collects

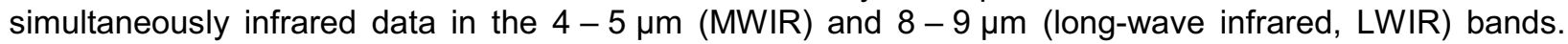
The demonstrator is based on a two-layer QWIP focal plane array with $384 \times 288 \times 2$ detector elements and $40 \mu \mathrm{m}$ pitch. Images are typically acquired with a frame rate of $100 \mathrm{~Hz}$ at $6.8 \mathrm{~ms}$ integration time and are stored as 14-bit digital data. Two optics were specially designed, first a dual field of view F/2 optics of $86 \mathrm{~mm}$ and $390 \mathrm{~mm}$ focal length, based on refractive and reflective components and second a refractive $100 \mathrm{~mm} \mathrm{~F} / 2$ optics.

The experimental assessment showed typical QWIP detectors with narrow bands and a low number of defective pixels. It shows a spectral crosstalk from the MWIR into the LWIR but not vice versa. The performance related to System Transfer Function (SiTF), Response, temporal and spatial Noise and Modulation Transfer Function (MTF) is similar in the MWIR and the LWIR. This is advantageous for image fusion techniques as well as for practical use, for example selecting the best band is thus not limited by the camera performance. Altogether the IR Dual-Band Camera demonstrator is comparable to the single band QWIPs and very appropriate for the analyses of dual-band techniques.

In addition to the experimental assessment of the demonstrator, image fusion techniques were presented. These techniques have the goal to show up the differences between the two bands in order to get new/additional information on the scene. In order to do so the bands have to be adapted somehow. For this purpose the "Apparent Temperature" method calibrates the two bands in apparent temperatures and ratio or subtraction reveals the differences. This method gives good results in laboratory tests and the first measurements in real military applications showed the possibility to improve observer performance for detection related tasks.

\section{ACKNOWLEDGEMENTS}

The IR-Dual-Band Camera demonstrator was supported by the German MOD under different BWB contracts. This support is gratefully acknowledged.

\section{REFERENCES}

1. M. Münzberg, R. Breiter, W. Cabanski, K. Hofmann, J. Wendler, J. Ziegler, R. Rehm, M. Walther, "Dual color IR detection modules, trends and applications", Proc. of SPIE Vol. 6542, 2007

2. H. Schneider, T. Maier, J. Fleissner, M. Walter, P. Koidl, G. Weimann, W. Cabanski, M. Finck, P. Menger, W. Rode, J. Ziegler, "Dual-band QWIP focal plane array for the second and third atmospheric windows", Infrared Physics \& technology 47 (2005), pp. 53-58

3. U. Adomeit, "Aspects Regarding the Experimental Assessment of Multispectral Thermal Imagers", FGAN-FOM Report FOM 2006/20, Ettlingen, 2006

4. "Experimental Assessment Parameters and Procedures for Characterization of Advanced Thermal Imagers", NATO RTO Technical Report RTO-TR-75(II) AC/323(SET-015)TP/21, February 2003

5. R. Driggers (Ed.), "Encyclopedia of Optical Engineering", Marcel Dekker Inc., New York, 2003

6. Arnold Goldberg, Theodore Fischer, Stephan Kennerly, William Beck, Vincent Ramirez, Karl Garner, "Laboratory and field imaging test results on single-color and dual-band QWIP focal plane arrays", Infrared Physics \& technology 42 (200), pp. 309-321

7. Dean Scribner, Penny Warren, Jon Schuler, Michael Satyshur, Melvin Kruer, "Infrared Color Vision: An Approach To Sensor Fusion", Optics \& Photonics News, August 1998, pp. 27-32

8. Peter R. N. Childs, "Practical Temperature Measurement", Butterworth Heinemann, Oxford, 2001

9. A. S. Tenney, "Radiation Ratio Thermometry", in D. P. DeWitt, Gene D. Nutter, "Theory and Practice of Radiation Thermometry", John Wiley /\& Sons, Inc., New York, 1988, pp. 459-494

10. George M. Williams, Archie Barter, "Dual-Band MWIR/LWIR Radiometer for Absolute Temperature Measurement", Proc. of SPIE Vol. 6025, 2006

11. U. Adomeit, W. Wittenstein, "Verfahren zur Inhomogenitätskorrektur und Kalibrierung von multispektralen Kameras“, FGAN-FOM Report OPT 2007/22, Ettlingen, 2007 